\title{
Diagnosis of Glaucoma using Artificial Neural Networks
}

\author{
H. P. Sathish Kumar \\ Research Scholar \\ Dravidian University \\ Kuppam, Andhra Pradesh
}

\author{
B. P. Mallikarjunaswamy \\ Ph.D, Professor \\ Dept. of Computer Science \& \\ Engg \\ Sri Siddhartha Institute of \\ Technology, Tumakuru-5
}

\author{
H. Venugopal \\ Ph.D, Professor \\ Dept. of Computer Science \& \\ Engg \\ Sri Siddhartha Institute of \\ Technology, Tumakuru-5
}

\begin{abstract}
Glaucoma is one of the severe eye disease according to the number of blindness causes in India and western Countries. Therefore the early detection, long-term monitoring of the patients and the decision about the appropriate therapy at the correct time point are serious tasks for the ophthalmologists and optometrists.

There are many diagnostic methods are available like, Fundal examination, perimetry OCT (Optical Coherence Tomography) Field analyzer and Tonometry to diagnose Glaucoma. Among these, Tonometry in the reliable and accurate method to measure the intra-ocular pressure of the eye. Which is the cause for Glaucoma.

The present research works in under taken to classify and diagnose such dreaded disease Glaucoma through Artificial Neural networks (ANNs) model. The ANN model adopted in multilayer feed forward networks and back propagation algorithm for classification. The present study considers 150 patients input data and output data for training of ANN networks, for testing of ANN, 50 patients input data is considered. The adopted ANN networks with topology 6-150-1 classified Glaucoma and non-glaucoma cases with an accuracy of $80 \%$.
\end{abstract}

\section{Keywords}

Glaucoma, Artificial Neural Networks, Tonometry, Optical Coherence Tomography, Single Layer Perceptron, Multi Layer Perceptron, Radial Basis Function networks, Kohonen's self-organizing feature map.

\section{INTRODUCTION}

The term glaucoma refers to a group of conditions that exhibit a loss of vision, often without symptoms. In glaucoma vision loss is caused by damage to the optic nerve, which transmits light signals from the retina to the brain, where they are translated into images. Figures 1 and 2 shows Normal and Glaucomatous Fundal Images.

Worldwide, there are an estimated 65-70 million cases of glaucoma. There are 3 million cases in the United States and 5 millions in India, but only one-half of those have been diagnosed. About $2 \%$ of people between the ages of 40 and 50 and $8 \%$ of those over 70 , have elevated intraocular pressure in one or both eyes.

Normal-tension glaucoma is more prevalent in people of Japanese ancestry and in those with a history of systemic heart disease. Family history of this disorder also increases the risk.

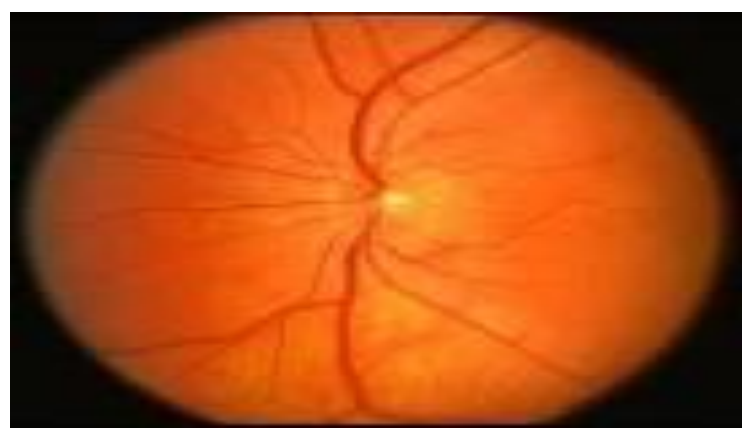

Fig.1 Normal Fundal Image

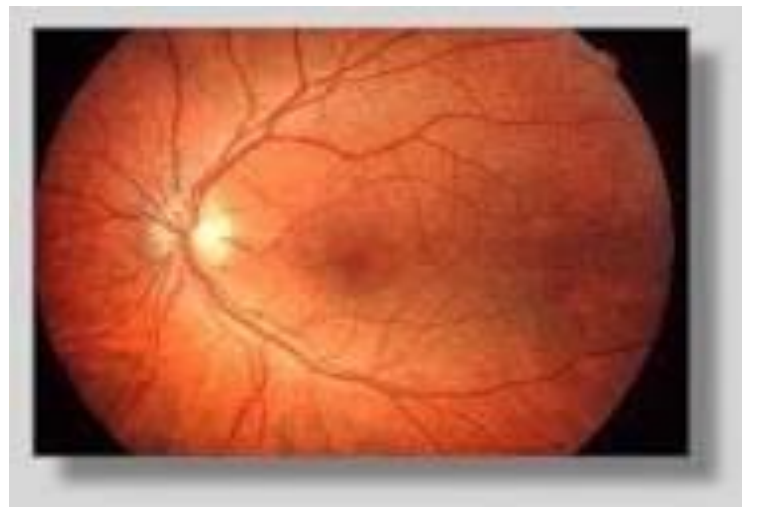

Fig.2 Glaucomatous Fundal Image

A neural network is a computational structure inspired by the study of biological neural processing. There are many types of neural networks like, Single Layer Perceptron (SLP), the Multi Layer Perceptron (MLP), Radial Basis Function (RBF) networks, Kohonen's self-organizing feature map networks. A multi layered feed-forward neural networks has layers or subgroups of processing element. A layer of processing elements makes independent computations on date that it receives and passes the results to another layer. The next layer may in turn make its independent computations and passes on the results to yet another layer. Finally a subgroup of one or more processing elements determines the output from the networks. Each processing element makes its computation based upon a weighted sum of its inputs. The first layer is the input layer and the last the output layer. The layers that are placed between the first and the last layers are the hidden layers. Figure 3 shows Multilayer perceptron Neural Network Architecture. 


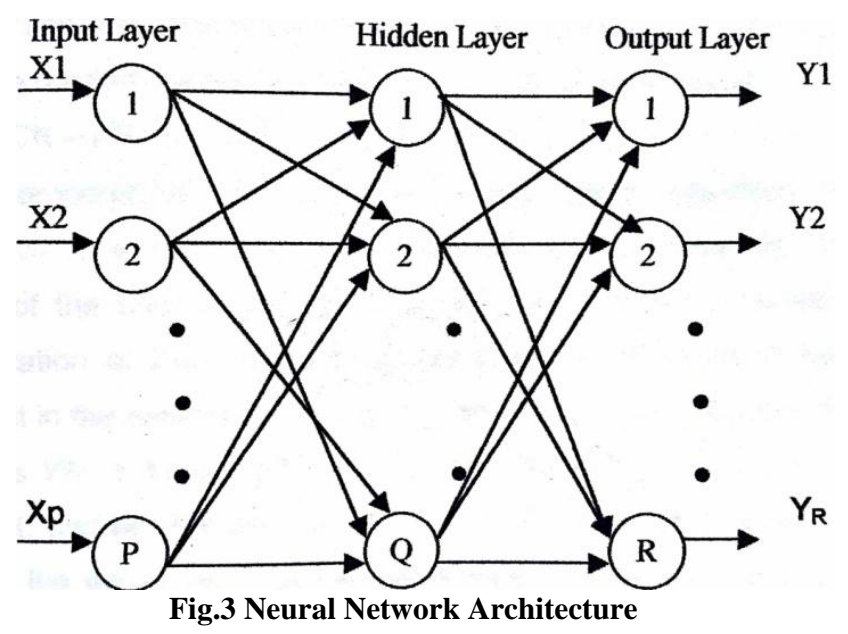

\section{ANN IN DIAGNOSIS OF}

\section{GLAUCOMA}

The design of the ANN classifier is based on the classification tree which is shown in figure 4(a). The motivation in modeling the classification tree is to introduce several decision stages ranging from rather crude decision like the 'normal'/'pathological' classification up to refined decision, and the 'questionable'/'probably' glaucomatous classification. Our approach in modeling the ANN perimetry-classifier is to design a specialized ANN for every decision level from the root to the leaves of the classification tree respectively as shown in figure 4(b). From the above classification tree the actual ANN for the present work is considered as follows.

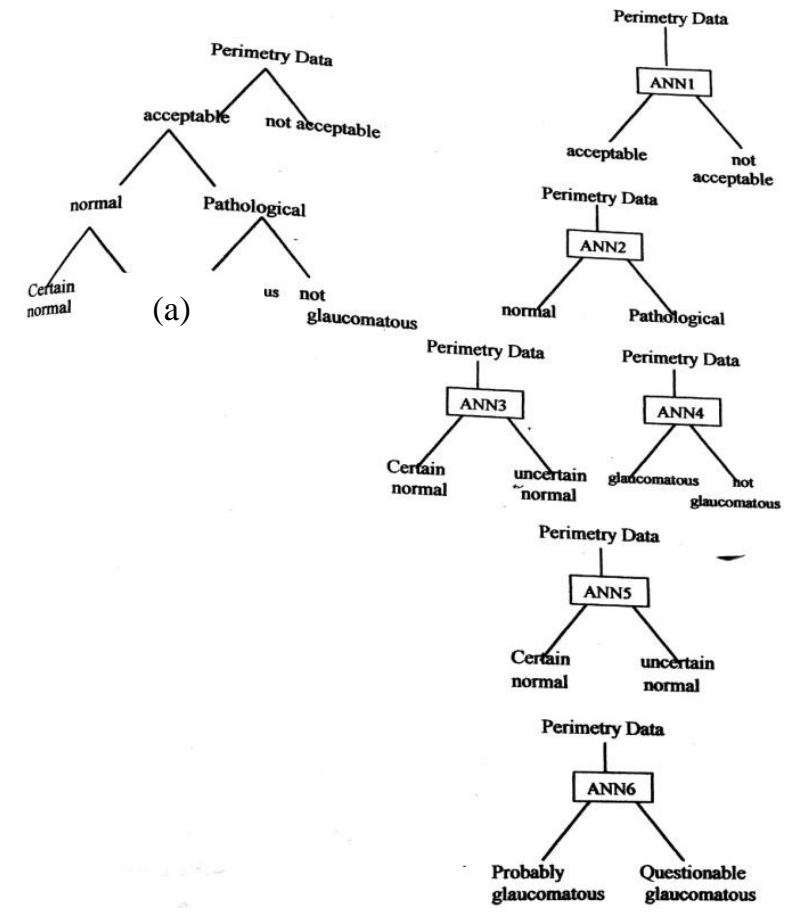

(b)

Fig.4 (a) Classification Tree, (b) ANN Classifier for Glaucoma disease classification

The specialization takes place in training every ANN exclusively on a part of the feature space which is defined by the set of perimetry samples according to their classification task. Furthermore the input dimensionality of every ANN is reduced by applying the method for dimensionality reduction, which is described below. Every ANN is consequently specialized on a decision boundary between two classes within the features space and the method for dimensionality reduction is applied to find a representation of the perimetry data, which makes the learning task for the ANNs as easy as possible. ANN Classifier Model for Glaucomatous and non Glaucomatous eye is shown in figure 5.

An advantage of the hierarchical classification scheme besides the specialization is that the whole feature space is covered which means that every possible perimetry can be correctly associated to a class of the hierarchy. Furthermore we have a refinement of the 'part of interest' in the feature space that is the class of glaucomatous perimetrices.

A perimetry data sample is classified by ANNs with topology 6-50-50-1 as shown in figure 6. Their output values are interpreted top down according to the classification tree.

\section{DATA PRE PROCESSING}

The goal in data pre-processing is to reduce the dimensionality of the perimetry data while improving the classification results of the applied ANNs. A method was developed for feature selection to receive interpretable results. Features selection is the determination of a subset of a set of features that optimizes a given criterion function. The data pre-processing step is applied on the training-database of the six specialized ANNs respectively to get an idea, which features of the perimetry data have good discrimination properties according to the special classification task. In a classification task, features that belong to the same class should be as similar as possible in contrast to features, which belong to different classes. The similarity of two featurevectors is metrics.

\section{RESULTS AND ANALYSIS}

The present research works adopts back propagation algorithm for ANN classifications as shown in Figures 5and 6. ANN classifier shown in figure 5 with topology 6-150-1 converges with less error for 6000 cycles as shown in figure 7 .

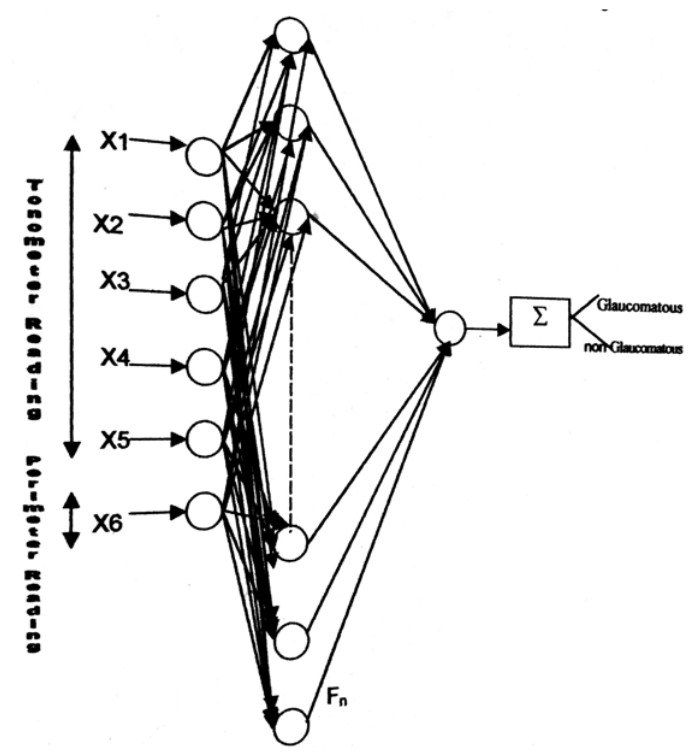

Fig.5 ANN Classifier Model with Topology 6-150-1 


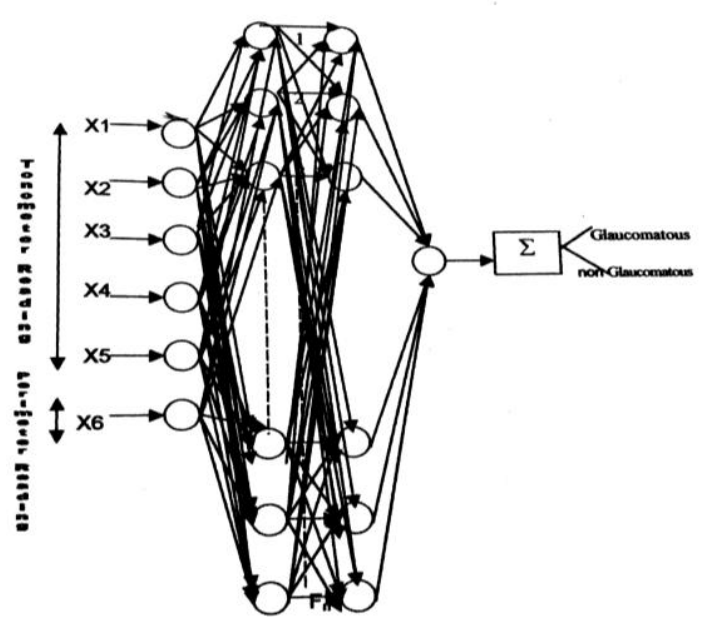

Fig.6 ANN Classifier with Topology 6-50-50-1

ANN classifier shown in figure 6 with topology 6-50-50-1 with 2000 cycles is more convergent giving relatively less error value of 0.004557 as shown in figure 8 . Compare to the ANN with two hidden layer topology is more accurate compare to first one.

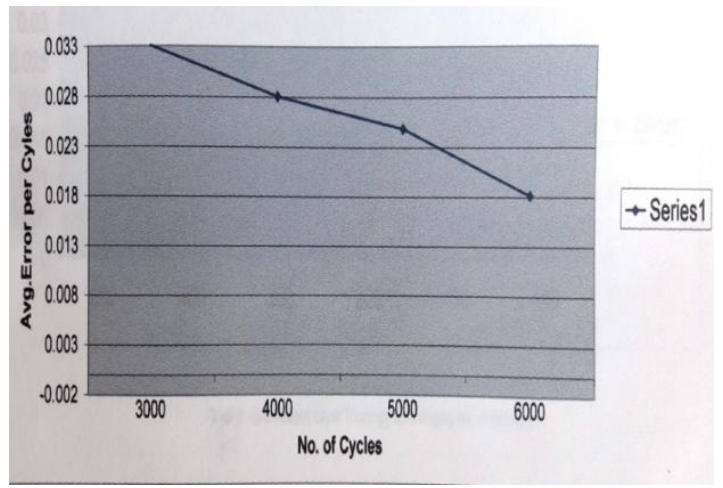

Fig. 7 Average Error Per Cycle v/s No. of Cycles

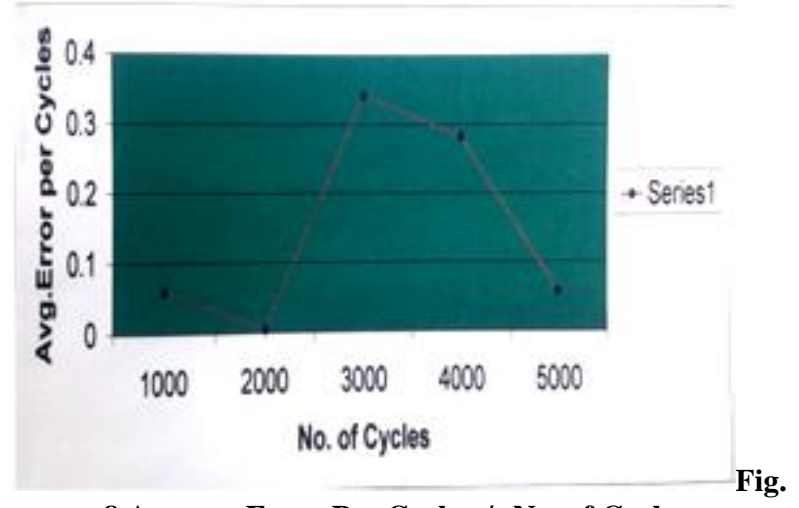

8 Average Error Per Cycle v/s No. of Cycles

\section{CONCLUSIONS}

The results of the present study shows neural networks models can be widely used for further medical diagnosis of glaucoma but however it needs further analysis and design using relevant soft computing techniques to develop expert systems for the diagnosis of glaucoma. The same analogy can be extended to the other branch of medical science for diagnostic purpose.

\section{REFERENCES}

[1] Gupta, H.V. et al., "Superior training of ANNs using weight-space partitioning", proceedings of IEEE conference on neural networks, Vol.3, Huston, Texas, 1997, pp. 1919-1923.

[2] Jacels M.Zurada, 'Introduction to Artificial Neural Systems", Jaico Publishing House, 2004.

[3] Simon Haykin, "Neural Networks, A Comprehensive foundation",Prentice-Hall, 2005.

[4] XIN YAO, 'Evolving Artificial Neural Networks", Proceedings of the IEEE, vol.87, No.9, September 1999.

[5] Sheeba-O, et al., "Glaucoma Detection Using Artificial Neural Networks", International Journal of Engineering and Technology, Vol. 6, No.2, Apr.2016, PP. 158-161.

[6] J.F Gullen, " The Pale Optic Disc", Some observation of G. European neuro- ophthalmologist in south east Asia, " Asian Journal of Opthalmology, Vol. 2, No.3, 2000.

[7] A. Peters, et al., "Diagnosis of Glaucoma by Indirect Classifiers" Methods of information in Medicine, 2003.

[8] Kurnitra Choudhary, Shamik Tiwari, 'ANN Glaucoma Detection Using Cup-to-Disks-Ratio and neuro ratinal Rim", International Journal of computer Applications, Volume 111, No 11, February 2015

[9] Pooja Chaudari, Prof. Girish A. kulakarni, "Using Artificial Neural Network to Detect Glaucoma with the help of cup to disk ratio", International Journal of Advanced Research in Electronics and Communication Engineering, Volume 5, Issue 7, July 2016.

[10] Hitesh Shirke, Dr.Nataraj Vijapur, "FPGA implementation of Glaucoma Detection Using Neural Networks", International Research Journal of Engineering and Technology, Vol. 04, Iissue 10, Oct 2017. 